\title{
Design of a 1-V 90-nm CMOS Folded Cascode LNA for Multi-Standard Applications
}

\author{
Edwin C. Becerra-Alvarez, Jose M. de la Rosa \\ Instituto de Microelectronica de Sevilla, IMSE-CNM \\ (CSIC/Universidad de Sevilla) \\ C/Americo Vespucio, 41092 Sevilla, SPAIN \\ E-mail: [edwin,jrosa]@imse-cnm.csic.es
}

\author{
Federico Sandoval \\ CINVESTAV-Unidad Guadalajara \\ Av. Cientifica No. 1145, 45010 \\ Col. El Bajio, Zapopan, Jalisco, MEXICO \\ E-mail: sandoval@gdl.cinvestav.mx
}

\begin{abstract}
This paper analyses the use of folded cascode Low Noise Amplifiers (LNAs) for the implementation of multistandard wireless transceivers. The proposed LNA consists of a two-stage topology made up of a folded cascode and simple-stage amplifiers that use NMOS-varactor based tuning networks to make the operating frequency continuously programmable. The circuit has been designed and implemented in a 90-nm CMOS technology in order to cope with the requirements of GSM, WCDMA, Bluetooth and WLAN (IEEE 802.11b/g) standards. Practical design issues are analysed, considering the effect of circuit parasitics associated to both the chip package and integrated inductors, capacitors and varactors; as well as technology parameter deviations. The circuit design is optimized using genetic algorithms to achieve the required specifications with adaptive power consumption. Layout-extracted simulation results demonstrate a correct operation of the proposed circuit, showing a continuous tuning of Noise Figure (NF) and $S$-parameters within the 1.85-2.48GHz band, featuring $\mathrm{NF}<3.8 \mathrm{~dB}, S_{21}>12 \mathrm{~dB}$ and IIP3 $>-12 \mathrm{dBm}$, with an adaptive power dissipation between $13.3 \mathrm{~mW}$ and $23.1 \mathrm{~mW}$ from a $1-\mathrm{V}$ supply voltage.
\end{abstract}

\section{INTRODUCTION}

The increasing number of personal wireless applications demands for Radio Frequency (RF) front-ends capable to handle different standard specifications, signal conditions and battery status [1]. The trend is towards a maximum hardware reuse, by making as many transceiver building blocks as possible, digitally programmable, reconfigurable and compatible with mainstream nanometer CMOS technologies [2].

Among other RF circuits, the design of the Low Noise Amplifier (LNA) is specially critical, due to its early position at the very beginning of the receiver chain, what makes this block a determinant factor in the overall system performance. The LNA has to simultaneously match the antenna and to amplify weak input signals with reduced noise contribution, high linearity and isolation from the rest of the receiver chain. This problem is aggravated in the case of multi-standard applications, in which LNAs must operate over different frequency ranges, whereas keeping reduced number of passive circuit elements to increase integration [1].

\footnotetext{
${ }^{1}$ This work has been supported by the Spanish Ministry of Science and Innovation (with support from the European Regional Development Fund) under contract TEC2007-67247-C02-01/MIC, and the Regional Council of Innovation, Science and Enterprise under contract TIC-2532.
}

The majority of multi-standard LNAs are based on the use of switchable passive networks to select the resonance frequency, thus preserving immunity to out-of-band interferers, although only one signal band is received at one time [3], [4]. Besides, the use of switches forces a discrete frequency selection and introduces parasitic switch-on resistances and capacitances, with the subsequent trade-off between linearity, noise and speed. This limitation can be partially solved by using concurrent multi-band LNAs [5], [6]; which allows a simultaneous reception of multiple signal bands without using switches. However, the spurs in one band may corrupt signals in other band due to the LNA non-linear operation.

A common issue in most reported multi-standard LNAs is the increase of the number of integrated passive elements (basically capacitors and inductors) as compared to their mono-standard counterparts, not offering a clear advantage with respect to using separate mono-standard LNAs [1], [2]. Moreover, most circuit topologies are based on stacked amplifiers (usually simple cascode) in either common-source or common-gate configuration, which are not very suited for low-voltage opperation. Recently, the use of folded cascode topology has been applied to the design of LNAs, although very little has been done for multi-standard applications [7].

This paper contributes to this topic and presents the design, layout implementation and electrical characterization of a reconfigurable two-stage folded cascode LNA in a 90-nm CMOS technology. The circuit is based on NMOS-varactor tuning networks to make the resonance frequency continuously programmable without needing any switches. An optimized design methodology has been used, that takes into account realistic equivalent circuit models for passive elements as well as the impact of package and technology parasitics. Post-layout simulations are shown to validate the presented approach.

\section{Proposed Reconfigurable Folded CAscode LNA}

Fig. 1 shows the schematic of the proposed reconfigurable LNA including the matching networks and biasing circuits. It consists of a two-stage topology with separate tuning networks. The input stage is a folded cascode amplifier formed by transistors $M_{n 1}$ and $M_{p 2}$ and inductor $L_{d}$, which resonates with interstage parasitic capacitance, thus providing the necessary high-impedance at the operation frequency. Inductor $L_{s}$ 


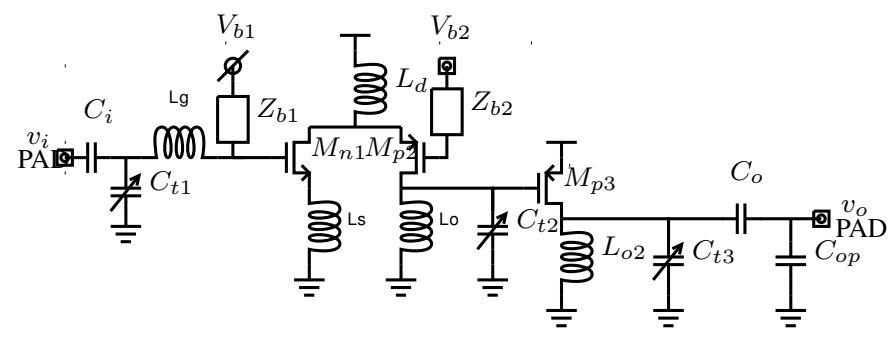

Fig. 1. Schematic of the proposed reconfigurable folded cascode LNA.

is used to implement source degeneration whereas $L_{o}$ is the load impedance. Folded cascode amplifier is very suited for low-voltage applications - since bias voltage can be reduced to one transistor overdrive - with reasonable good linearity, stability and Noise Figure (NF) [7]. Thus, in order to get the required forward gain, $S_{21}$, an additional amplifier stage formed by PMOS transistor $M_{p 3}$ and load inductor $L_{o 2}-$ is used. The circuit is biased by dc voltages $V_{b 1}$ and $V_{b 2}$, which are respectively connected at the gates of $M_{n 1}$ and $M_{p 2}$ through the impedances $Z_{b 1}$ and $Z_{b 2}$ - implemented by diodeconected MOS transistors.

As the LNA is fully integrated as a stand-alone circuit, a termination of $50 \Omega$ is needed at both the input and the output terminals connected at the bonding PADs (see Fig. 1). For this purpose, $C_{i}$ and $L_{g}$ are used to provide the required input impedance, $Z_{i n}$, whereas decoupling capacitors $C_{o}$ and $C_{o p}$ are used at the output node. The tuning mechanism of the LNA is achieved by varying the resonance frequencies of the different passive networks, through accumulation NMOS varactors, $C_{t 1-3}$.

\section{A. Effect of Circuit Parasitics}

The schematic in Fig. 1 assumes ideal circuit elements, particularly integrated capacitors, varactors and inductors. In practice, circuit parasitics cause these elements to behave as RLC networks that may severely degrade the performance of the LNA [8]. This is specially important in the case of reconfigurable/adaptive RF circuits like the one proposed in this paper, because of the many different specifications to be covered by the same circuit.

In order to evaluate the impact of circuit parasitics, the proposed LNA was analyzed by replacing every inductor and varactor in Fig. 1 with the equivalent circuit shown in Fig. 2 [8], [9]. In the case of the inductor (Fig. 2(a)), the nominal inductance is given by $L_{s 1}+L_{s 2}$, whereas the remaining elements are circuit parasitics: $C_{o 1-3}$ and $R_{b 1-3}$ are respectively substrate capacitances and resistances; $R_{s 1-2}$ are series resistances and $C_{s 1-2}$ are embedded coupling capacitances. In the case of varactors (Fig. 2(b)), $C_{p}$ is the nominal capacitance and $C_{c}$ is the variable voltage controlled capacitance, whereas the rest of circuit elements are parasitics, with $L_{1-2}$ and $R_{p 1-2}$ being series inductances and resistances, respectively, and $R_{w}$ is the well parasitic resistance.

\section{B. Circuit analysis}

Replacing the inductors and varactors in Fig. 1 with the RLC equivalent circuits shown in Fig. 2, it can be shown that,

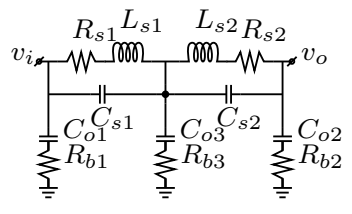

(a)

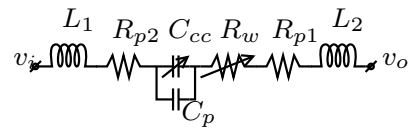

(b)
Fig. 2. Equivalent RLC circuit for (a) inductors and (b) varactors.

after some approximations, the input impedance $\left(Z_{i}\right)$ and the noise factor $(F)$ of the proposed LNA are respectively given by:

$$
\begin{aligned}
& Z_{i}(s) \simeq \frac{Z_{i 2}\left(g_{m n 1}+s C_{g s n 1}\right)+Z_{i 4}}{s C_{i}+s C_{i} Z_{i 2}\left(g_{m n 1}+s C_{g s n 1}\right)+s Z_{i 1} Z_{i 4}} \\
& F \simeq 1+\left(\frac{1}{g_{m n 1} R_{R F}}\right)\left(\frac{R_{R F}-Z_{i}}{s C_{i} Z_{b 1} Z_{i}}\right)^{2} \cdot \\
& \quad\left(\frac{\gamma}{\alpha}-\frac{s^{2} C_{g s n 1}^{2} Z_{1}^{2} \alpha \delta}{5}+\left(2|c| s C_{g s n 1} Z_{1}\right) \sqrt{\frac{\gamma \delta}{5}}\right)
\end{aligned}
$$

where

$$
\begin{aligned}
& Z_{i 1}(s) \simeq \frac{C_{c t 1}+C_{p t 1}}{1+2 s R_{p 1 t 1}\left(C_{c t 1}+C_{p t 1}\right)} \\
& Z_{i 2}(s) \simeq \frac{4\left(R_{s 1 s}+s L_{s 1 s}\right)\left(2+5 s C_{o 3 s} R_{b 3 s}\right)}{4+s C_{o 3 s}\left(10 R_{b 3 s}+3\left(R_{s 1 s}+s L_{s 1 s}\right)\right)} \\
& Z_{i 3}(s) \simeq \frac{4\left(R_{s 1 g}+s L_{s 1 g}\right)\left(2+5 s C_{o 3 g} R_{b 3 g}\right)}{4+s C_{o 3 g}\left(10 R_{b 3 g}+3\left(R_{s 1 g}+s L_{s 1 g}\right)\right)}+Z_{i 2} \\
& Z_{i 4}(s) \simeq s C_{i} Z_{b 1}\left(1+g_{m n 1} Z_{i 2}+s C_{g s n 1} Z_{i 3}\right) \\
& \omega_{0} \simeq \quad Z_{1}=Z_{b 1}+Z_{i 2}+s C_{i t 1} Z_{i 3} Z_{b 1} \\
& \sqrt{\left(L_{g}+L_{s}\right) C_{g s n 1}}
\end{aligned}
$$

with $c$ being the correlation factor; $\delta$ and $\gamma$ are technology parameters; $R_{R F}$ is the RF source resistance; $\alpha=g_{m n 1} / g_{d s n 1}$; and $g_{d s n 1}, g_{m n 1}$ and $C_{g s n 1}$ are respectively the small-signal drain-source conductance, transconductance and gate-source capacitance of $M_{n 1}$. Parasitic resistors, capacitors and inductors $\left(R_{p}, R_{s}, C_{p}\right.$, etc.) are labelled accordingly to the name of their associated varactor/inductor in Fig. 1.

The real part of $Z_{i}$ is chosen to be equal to $R_{R F}$ - usually $50 \Omega$ - in the band of interest, $1.85-2.48 \mathrm{GHz}$ in this case. In order to achieve proper impedance matching within this band, inductor $L_{g}$ and varactor $C_{t 1}$ are properly sized to get the required frequency tuning. This is illustrated in Fig. 3 by showing the trajectories of the values of these elements towards impedance matching within the band of interest, with $Z_{s i}$ being the input impedance seen at the right of $L_{g}$.

The same tuning mechanism - based on the use of varactors $C_{t 2,3}$ - is used to vary the output impedance and the voltage gain, approximately given by: 


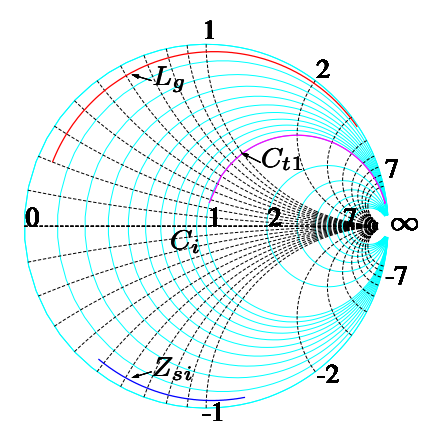

Fig. 3. Input impedance matching trajectories in the Smith chart.

$$
\begin{aligned}
A_{v} \simeq & \left(\frac{g_{m n 1} Z_{v 2} Z_{v 3}\left(g_{m p 3}+s C_{g s p 2} g_{d s p 2} Z_{b 2}\right)}{g_{m p 2} Z_{v 2}+n_{2}+n_{1}\left(1+s C_{g s p 2}\left(Z_{b 2}+Z_{v 2}\right)\right)}\right) . \\
& \left(\frac{g_{m p 3} R_{L} Z_{o 2}}{R_{L}+\left(1+s R_{L} Z_{o 1}\right) Z_{o 2}}\right)
\end{aligned}
$$

where

$$
\begin{aligned}
Z_{o 1}(s) & \simeq \frac{C_{c t 3}+C_{p t 3}}{1+2 s R_{p 1 t 3}\left(C_{c t 3}+C_{p t 3}\right)} \\
Z_{o 2}(s) & \simeq \frac{4\left(R_{s 1 o 2}+s L_{s 1 o 2}\right)\left(2+5 s C_{o 3 o 2} R_{b 3 o 2}\right)}{4+s C_{o 3 o 2}\left(10 R_{b 3 o 2}+3\left(R_{s 1 o 2}+s L_{s 1 o 2}\right)\right)} \\
Z_{v 1}(s) & \simeq \frac{C_{c t 2}+C_{p t 2}}{1+2 s R_{p 1 t 2}\left(C_{c t 2}+C_{p t 2}\right)} \\
Z_{v 2}(s) & \simeq \frac{4\left(R_{s 1 d}+s L_{s 1 d}\right)\left(2+5 s C_{o 3 d} R_{b 3 d}\right)}{4+s C_{o 3 d}\left(10 R_{b 3 d}+3\left(R_{s 1 d}+s L_{s 1 d}\right)\right)} \\
Z_{v 3}(s) & \simeq \frac{4\left(R_{s 1 o}+s L_{s 1 o}\right)\left(2+5 s C_{o 3 o} R_{b 3 o}\right)}{4+s C_{o 3 o}\left(10 R_{b 3 o}+3\left(R_{s 1 o}+s L_{s 1 o}\right)\right)} \\
n_{1}=1 & +s Z_{v 1} Z_{v 3} ; \quad n_{2}=g_{d s p 2} Z_{v 3}\left(1+s C_{g s p 2} Z_{b 2}\right)
\end{aligned}
$$

\section{Design Methodology}

The proposed LNA has been designed to fulfill the requirements of four wireless standards: GSM, Bluetooth, WCDMA and WLAN (IEEE $802.11 \mathrm{~b} / \mathrm{g}$ ), including continuously-tuning operation within the band of interest. In order to cope with the different sets of specifications, the design equations described in previous section were used to find out an initial set of design parameters, as well as proper biasing to guarantee all transistors operating in the saturation region.

The initial design parameters are used as start point of an optimized design process, which combines electrical simulations using Cadence SpectreRF with genetic-based algorithms [10] in order to find out the best design parameters that fulfill the required specifications with minimum power consumption. For that purpose, the circuit in Fig. 4 was used. This circuit includes the package and the external components to be included in the PCB. A $4 \mathrm{mmx} 4 \mathrm{~mm} 12$-pin QFN plastic package has been used. This package has been modeled using Cadence PKG tool in order to take into account their associated parasitics during the design process.

Fig. 5(a) shows the flow diagram of the optimization process, where after a random variation of one of the design

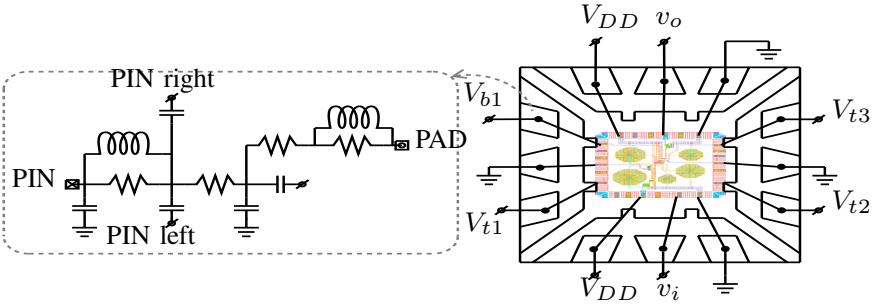

Fig. 4. Equivalent circuit including chip-package parasitics.

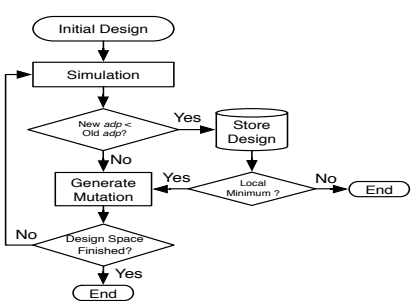

(a)

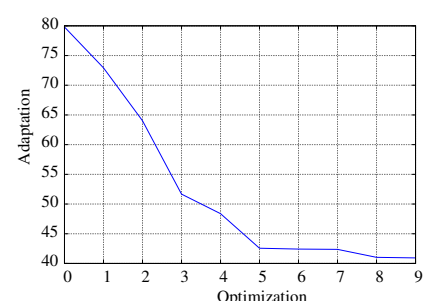

(b)
Fig. 5. Optimization procedure: (a) Flow diagram. (b) Adaptation function.

parameters - referred to as mutation - a set of simulations are performed to evaluate the performance of the LNA. The data extracted from simulations are used to evaluate a cost function - called adaptation - defined as:

$$
\begin{aligned}
a d p \simeq & \left|S_{11 i}-S_{11 r}\right|+\left|S_{21 i}-S_{21 r}\right|+\left|S_{22 i}-S_{22 r}\right| \\
& +\left|N F_{i}-N F_{r}\right|+\left|f_{c S 11 i}-f_{c S 11 r}\right| \\
& +\left|f_{c S 21 i}-f_{c S 21 r}\right|+\left|f_{c S 22 i}-f_{c S 22 r}\right| \\
& +\left|f_{c N F i}-f_{c N F r}\right|+\left(S_{11 \max }-S_{11 \min }\right) \\
& +\left(S_{21 \max }-S_{21 \min }\right)+\left(S_{22 \max }-S_{22 \min }\right) \\
& +\left(N F_{\max }-N F_{\min }\right)
\end{aligned}
$$

where the subscripts $i$ and $r$ denote the ideal and real values of a given parameter; and $f_{c}$ stands for the central frequency of the frequency response of that parameter. For instance, $f_{c N F i}$ is the central frequency of the ideal NF function. Once $a d p$ is computed for a given set of design parameters, a new mutation is generated and the process is repeated again until a minimum value of adaptation is found as illustrated in Fig. 5(b). In order to find out a global minimum, local minima are stored in a best-designs table, which remembers these minima in order to avoid them in ulterior mutations.

The outcome of the design procedure described above is the sizing and biasing of the LNA - summarized in Table I. As stated above, the performance of the circuit is adapted to the different standards specifications by varying the values of varactor capacitances - also shown in Table I.

TABLE I

LNA SIZING

\begin{tabular}{|c|c|cc|}
\hline Capacitors (pF) & Inductors (nH) & Transistors & W/L $(\mu \mathbf{m} / \mu \mathbf{m})$ \\
\hline$C_{i}=1.9 ; C_{o}=2.5$ & $L_{d}=3.13$ & $\mathrm{M}_{n 1}$ & $113 / 0.3$ \\
\hline$C_{O p}=0.48$ & $L_{g}=5.60$ & $\mathrm{M}_{p 2}$ & $455 / 0.3$ \\
\hline$C_{t 1}=(0.08-0.33)$ & $L_{s}=1.23$ & $\mathrm{M}_{p 3}$ & $32 / 0.3$ \\
\hline$C_{t 2}=(0.08-0.31)$ & $L_{o}=4.60$ & \multicolumn{2}{|c}{} \\
\cline { 1 - 2 }$C_{t 3}=(0.10-0.35)$ & $L_{o 2}=1.95$ & & \\
\cline { 1 - 2 } &
\end{tabular}




\section{LAyout AND Simulation Results}

The LNA has been implemented using a 90-nm CMOS technology with a single $1-\mathrm{V}$ supply voltage. Fig. 6 shows the layout of the chip highlighting their main parts, namely integrated inductors, capacitors, NMOS varactors and CMOS active area. Integrated inductors have octagonal shape with a patterned ground shield. Input/output capacitors are implemented by M-O-M structures, which are based on the combination of stacked and finger metal-metal capacitors. All pads are ElectroStatic Discharged (ESD) protected. The die area, including pads, is $0.64 \mathrm{~mm}^{2}$.

Fig. 7(a) represents NF vs. input frequency for all standards under study. The overall minimum value of $\mathrm{NF}$ is $3.1 \mathrm{~dB}$, obtained at $2.44 \mathrm{GHz}$, which corresponds to the WLAN operation mode. Fig. 7(b)-(d) show respectively $S_{21}, S_{11}$ and $S_{22}$. The minimum value of $S_{21}$ within the band of interest is above $12 \mathrm{~dB}$, corresponding to WCDMA, whereas $S_{11}$ and $S_{22}$ are below $-8.5 \mathrm{~dB}$ for all standards.

The operation of the circuit can be continuously tuned within the band of interest. This feature - illustrated in Fig. 8 for $S_{21}$ - is a direct consequence of using NMOS varactors in the resonance tuning networks, which provides approximately $700-\mathrm{MHz}$ tuning range. Indeed, this is a peculiarity of the presented chip as compared to previously reported multi-standard LNAs - mostly based on using switchable LC tanks to select the operating frequency in a discrete way and using varactors to fine tuning the selected band. The difference in the presented design is that it achieves a coarse continuous frequency tuning capability, just using varactors. Finally, Table II sums up the simulated performance of the LNA by showing the values of the different performance figures for each standard.

\section{CONCLUSIONS}

The design and electrical implementation of a multistandard 1-V 90-nm CMOS folded cascode LNA has been presented. The use of NMOS-varactor based tuning networks allows the amplifier to adapt its performance to the specifications of different wireless standards. An optimizationbased design methodology has been used to find out the target requirements with adaptive power dissipation. Layoutextracted simulations including packaging circuit parasitics demonstrate a correct performance of the circuit.

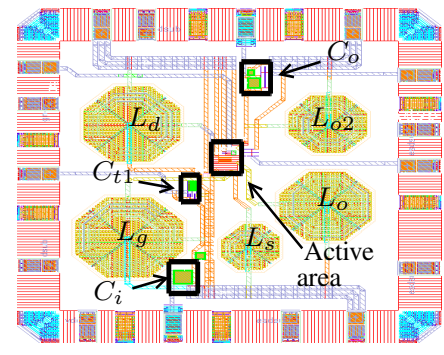

Fig. 6. Layout of the LNA. (a)

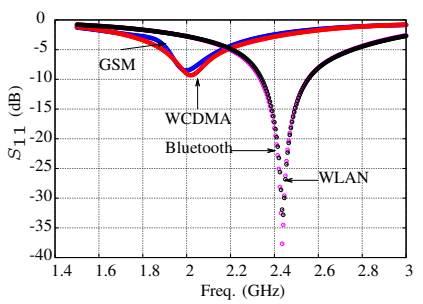

(c)

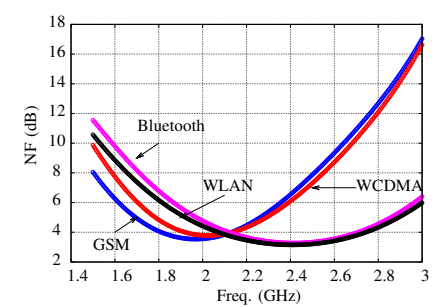

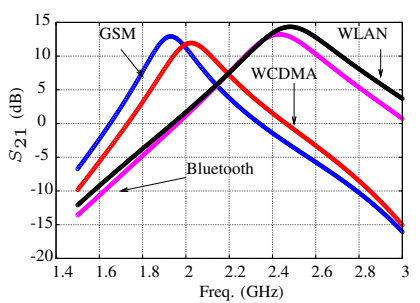

(b)

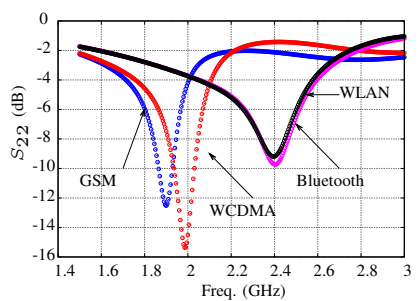

(d)
Fig. 7. Simulated NF and S-parameters.

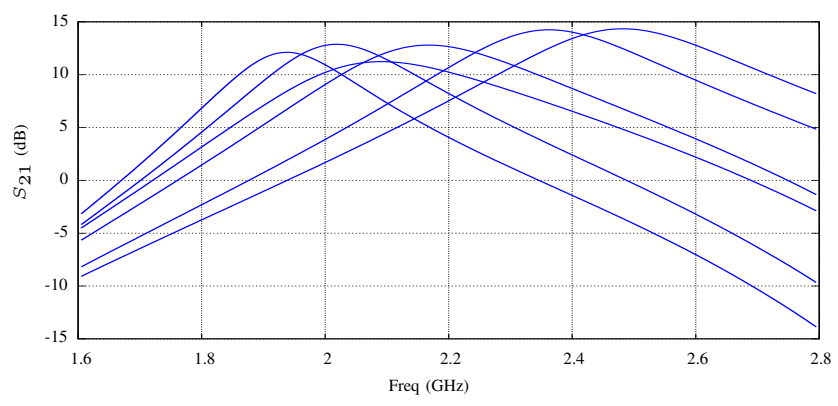

Fig. 8. Illustrating the reconfiguration of $S_{21}$.

\section{REFERENCES}

[1] M. Brandolini et al., "Toward Multistandard Mobile Terminals - Fully Integrated Receivers Requirements and Architectures," IEEE Trans. on Microwave Theory and Techniques, vol. 53, pp. 1026-1038, 2005.

[2] P.-I. Mak et al., "Transceiver Architecture Selection: Review, State-ofthe-Art Survey and Case Study," IEEE Circ. and Syst. Mag., 2007.

[3] V. Vidojkovic et al., "Fully-integrated DECT/Bluetooth multi-band LNA in 0.18um CMOS," Proc. of IEEE ISCAS, pp. 565-568, 2004.

[4] Y. Koolivand et al., "A new technique for design CMOS LNA for multistandard receivers," Proc. of IEEE ISCAS, vol. 4, pp. 3231-3234, 2005.

[5] H. Hashemi et al., "Concurrent multiband low-noise amplifiers-theory, design, and applications," IEEE Transactions on Microwave Theory and Techniques, vol. 50, pp. 288-301, Jan 2002.

[6] C.-W. Ang et al., "A Multi-band CMOS Low Noise Amplifier for Multistandard Wireless Receivers," Proc. of IEEE ISCAS, pp. 2802-2805, 2007.

[7] H.-H. Hsieh et al., "Gain-Enhancement Techniques for CMOS Folded Cascode LNAs at Low-Voltage Operations," IEEE Transactions on Microwave Theory and Techniques, vol. 56, no. 8, pp. 1807-1816, 2008.

[8] T. H. Lee, The Design of CMOS Radio-Frequency Integrated Circuits. Cambrige University Press, 2 ed., 2004.

[9] P. Vizmuller, RF Design Guide. Artech House, 1995.

[10] M. D. Vose, The Simple Genetic Algorithm: Foundations and Theory. The MIT Press, 1999.

TABLE II

LNA PERFORMANCE

\begin{tabular}{|c|c|c|c|c|c|}
\hline Standard & IIP3 $\mathbf{( d B m})$ & $\mathbf{N F}(\mathbf{d B})$ & $S_{21}(\mathbf{d B})$ & $S_{11}(\mathbf{d B})$ & $P_{D C}(\mathbf{m W})$ \\
\hline GSM & -9.9 & 3.5 & 12.9 & -8.5 & 23.1 \\
\hline WCDMA & -11.9 & 3.8 & 12.0 & -9.3 & 16.0 \\
\hline Bluetooth & -10.5 & 3.3 & 13.2 & -15.2 & 13.3 \\
\hline WLAN & -11.6 & 3.1 & 14.3 & -15.4 & 16.0 \\
\hline
\end{tabular}

